\title{
Oblique Corrections in the MSSM at One-Loop-Part I: Scalars
}

\author{
Yao Yu and Sibo Zheng \\ Department of Physics, Chongqing University, Chongqing 401331, China \\ Correspondence should be addressed to Sibo Zheng; sibozheng.zju@gmail.com
}

Received 17 July 2013; Accepted 18 September 2013

Academic Editor: Elias C. Vagenas

Copyright (c) 2013 Y. Yu and S. Zheng. This is an open access article distributed under the Creative Commons Attribution License, which permits unrestricted use, distribution, and reproduction in any medium, provided the original work is properly cited.

\begin{abstract}
This paper is devoted to reconsider the one-loop oblique corrections arising from the scalar superpartners in the MSSM, that is, the squarks, the sleptons, and the scalars in Higgs sector. We explicitly present the complete one-loop forms of self-energy corrections to the gauge bosons of SM electroweak gauge groups, as well as their descendants the $S, T$, and $U$ parameters, which can be directly applied to constrain the parameter space of the MSSM. Our results about one-loop self energies are found to agree with Drees et al. (1992) and Pierce et al. (1997). Nevertheless, the $S, T$, and $U$ parameters are not in agreement with Dobado et al. (1999).
\end{abstract}

\section{Introduction}

With LHC keeping running on searches of the standard model (SM) Higgs with mass around $125 \mathrm{GeV}[1,2]$, we are at the dawn when studies of Higgs physics become a field of the precise test, and we find out whether low-scale supersymmetry is going to show up.

Among frameworks in which the implication of the recent LHC results in new physics are explored, the electroweak precise test always stands as an important way for studying phenomenology of Higgs physics. The logic is that SM (naturally including the Higgs particle $h$ ) and its extensions such as supersymmetric SM can be analyzed through considering the quantum corrections, more concretely oblique corrections $[3,4]$, in these theories to the electroweak precise test observables. In SM, the oblique corrections mainly depend on the Higgs particle mass $m_{h}$ and top quark mass, it follows that electroweak precise test provides useful constraint on $m_{h}$ [5]. For example, it is very successful in analyzing technicolor models [4].

This method can be similarly applied in SUSY models, from which the masses of supersymmetric particles can be constrained (see, e.g., [6]). But this method is not very efficient due to some reasons. One is that there are so many mass parameters in SUSY models. Another is that the bounds on these masses are not yet sufficient before the running of the LHC collider [7-9]. So even in the minimal supersymmetric standard model (MSSM), it is impossible to make firm claims.
In order to extract useful information, as discussed in the literature, various limits such as highly degenerate $[5,10]$ or super heavy masses [11-13] of supersymmetric particles are considered in SUSY models.

However, these approximations are too simple to be suitable when the present LHC data is incorporated into the MSSM. Also, it can be verified that the analytic results related to our discussion usually are not consistent in the literature. Due to these observations, we should ascertain the oblique corrections in the MSSM first. In this paper, we will address this issue by using the two-component formalism of Lagrangian for supersymmetric particles under the electroweak group. The main outcomes are two fold. At first, the results we obtain can be applied to the most general cases without taking any assumptions about the quark- and lepton scalar masses. Moreover, our results cannot be reduced to those of [11] when we use the same limit as that the authors took. The difference is probably due to the missing or the over counting of Feynman loops involving heavy scalars as the internal lines.

We want to emphasize that while this work is being prepared, the LHC results $[1,2]$ indicate that its main task has been transformed from discovery to precise tests of the SM-like Higgs and discovery of supersymmetry. The oblique corrections to electroweak observables can still serve as a window to explore the parameter space of superpartners' masses by combining the data collected by the LHC and other 
colliders. The implications to MSSM and NMSSM along this line will be addressed elsewhere [14].

The paper is organized as follows. In Section 2, we derive the bosonic oblique corrections in the MSSM. We divide the task into three parts, that is, the squark sector, the slepton sector, and the Higgs sector. We will consider the fermionic contributions in the paper [15]. In Section 3, we then make preliminary checks on the results presented in Section 2 in terms of the fact that the radiative corrections to electroweak mixing angle is finite. In Section 4, we present the bosonic results of $S, T$, and $U$ parameters in the MSSM. The property that these parameters are also finite is more stringent examination on the results than the one in Section 3. In Section 5, we conclude and discuss our main results. We find that our results about one-loop self energies are found to agree with $[6,16]$. Nevertheless, the $S, T$, and $U$ parameters do not math with those of [11]. In the appendix explicit expressions for the functionals related to one-loop graphs are presented. We would like to emphasize that the on-shell renormalization scheme is used throughout this paper.

\section{One-Loop Bosonic Contributions}

Since we deal with the analytic calculation of one-loop self energy of SM electroweak gauge bosons, it is convenient to use the two-component formalism both for the supersymmetric scalars and fermions.

2.1. Lagrangian for Electroweak Scalar Doublets. To derive the Feynman rules, we refer to the Lagrangian for scalar doublets $\Phi^{T}=\left(\phi_{1}, \phi_{2}\right)$ under the representation of electroweak gauge group, which can be written as

$$
\mathscr{L}_{H} \sim\left(\vec{D}_{\nu} \Phi\right)^{\dagger}\left(\vec{D}^{\nu} \Phi\right)=\Phi^{\dagger} \overleftarrow{D}_{\nu} \vec{D}^{v} \Phi
$$

with the definitions

$$
\vec{D}_{\nu} \Phi=\left(\begin{array}{cc}
\vec{\partial}_{v}-i\left(\frac{e}{2 s c}-\frac{e s}{c} Q_{1}\right) Z_{v}-i e Q_{1} A_{v} & -i \frac{e}{\sqrt{2} s} W_{v}^{+} \\
-i \frac{e}{\sqrt{2} s} W_{v}^{-} & \vec{\partial}_{v}+i\left(\frac{e}{2 s c}+\frac{e s}{c} Q_{2}\right) Z_{v}-i e Q_{2} A_{v}
\end{array}\right) \Phi
$$

Here $Q_{1}$ and $Q_{2}$ represent the $Y$-charges of up-type $\phi_{1}$, and down-type $\phi_{2}$, respectively. And

$$
\Phi^{\dagger} \overleftarrow{D}_{v}=\Phi^{\dagger}\left(\begin{array}{cc}
\overleftarrow{\partial}_{v}+i\left(\frac{e}{2 s c}-\frac{e s}{c} Q_{1}\right) Z_{v}+i e Q_{1} A_{v} & i \frac{e}{\sqrt{2} s} W_{v}^{+} \\
i \frac{e}{\sqrt{2} s} W_{v}^{-} & \overleftarrow{\partial}_{v}-i\left(\frac{e}{2 s c}+\frac{e s}{c} Q_{2}\right) Z_{v}+i e Q_{2} A_{v}
\end{array}\right)
$$

where parameters $s=\sin \theta_{W}$ and $c=\cos \theta_{W}$.

For EW singlet scalars $S$ such as right-hand squarks and sleptons, the Lagrangian is given by

$$
\begin{aligned}
\mathscr{L}_{H} \sim\left(D_{\nu} S\right)^{\dagger}\left(D^{\nu} S\right)= & S^{*}\left(\overleftarrow{\partial}_{\nu}-\underset{c}{\left.i-e Q Z_{v}+i e Q A_{\nu}\right)}\right. \\
& \times\left(\vec{\partial}^{v}+\underset{c}{\left.i \underset{c}{s} e Q Z^{v}-i e Q A^{\nu}\right) S}\right.
\end{aligned}
$$

Here, $Q$ denotes the $Y$-charge of $S$ field.

From (1) to (4), we can derive the Feynman rules for MSSM scalars (see, e.g., [17]). In what follows, we take the 't Hooft-Feynman gauge for nonabelian gauge fields involved. It turns out that there are three types of graphs as shown in Figure 1 needed to be considered.
2.2. Squark Sector. The squark contributions are composed of those coming from three-generation left-hand squarks $\tilde{q}_{L i}=\left(\widetilde{u}_{i}, \widetilde{d}_{i}\right)$ and their right-hand $\tilde{u}_{R i}, \widetilde{d}_{R i}$. According to the Lagrangian (1), we find that there are two types of Feynman diagrams that contribute to the one-loop self-energy of vector bosons, as shown in Figures 1(a) and 1(b). (In the next subsection there will be an extra Feynman diagram that needs to be counted due to the Higgs VEVs.) We find (We have neglected $1 / 16$ factor in each $\Pi^{V V^{\prime}}$ in this section, which will be restored in Section 4.)

$$
\begin{aligned}
\Pi_{L}^{\gamma \gamma}\left(q^{2}\right)=N_{c} \frac{e^{2}}{9 \pi^{2}} \sum_{i=1,2,3}[ & -16 A\left(q^{2} ; m_{\widetilde{u}_{i}}^{2}, m_{\tilde{u}_{i}}^{2}\right) \\
& -4 A\left(q^{2} ; m_{\tilde{d}_{i}}^{2}, m_{\tilde{d}_{i}}^{2}\right) \\
& \left.+8 a\left(m_{\tilde{u}_{i}}^{2}\right)+2 a\left(m_{\tilde{d}_{i}}^{2}\right)\right],
\end{aligned}
$$




$$
\begin{aligned}
& \Pi_{L}^{Z Z}\left(p^{2}\right)=N_{c} \frac{1}{\pi^{2}} \sum_{i=1,2,3}\left[-4\left(\frac{e}{2 s c}-\frac{2 e s}{3 c}\right)^{2}\right. \\
& \times A\left(q^{2} ; m_{\widetilde{u}_{i}}^{2}, m_{\widetilde{u}_{i}}^{2}\right) \\
& -4\left(\frac{e}{2 s c}-\frac{e s}{3 c}\right)^{2} \\
& \times A\left(q^{2} ; m_{\tilde{d}_{i}}^{2}, m_{\tilde{d}_{i}}^{2}\right) \\
& +2\left(\frac{e}{2 s c}-\frac{2 e s}{3 c}\right)^{2} a\left(m_{\tilde{u}_{i}}^{2}\right) \\
& \left.+2\left(\frac{e}{2 s c}-\frac{e s}{3 c}\right)^{2} a\left(m_{\tilde{d}_{i}}^{2}\right)\right], \\
& \Pi_{L}^{\gamma Z}\left(q^{2}\right)=N_{c} \frac{e}{3 \pi^{2}} \sum_{i=1,2.3}\left[-8\left(\frac{e}{2 s c}-\frac{2 e s}{3 c}\right)\right. \\
& \times A\left(q^{2} ; m_{\tilde{u}_{i}}^{2}, m_{\tilde{u}_{i}}^{2}\right) \\
& -4\left(\frac{e}{2 s c}-\frac{e s}{3 c}\right) \\
& \times A\left(q^{2} ; m_{\tilde{d}_{i}}^{2}, m_{\tilde{d}_{i}}^{2}\right) \\
& +4\left(\frac{e}{2 s c}-\frac{2 e s}{3 c}\right) a\left(m_{\tilde{u}_{i}}^{2}\right) \\
& \left.+4\left(\frac{e}{2 s c}-\frac{e s}{3 c}\right) a\left(m_{\tilde{d}_{i}}^{2}\right)\right], \\
& \Pi_{L}^{W W}\left(q^{2}\right)=N_{c} \frac{1}{2 \pi^{2}} \frac{e^{2}}{s^{2}} \sum_{i=1,2.3}\left[\left(a\left(m_{\tilde{u}_{i}}^{2}\right)+a\left(m_{\tilde{d}_{i}}^{2}\right)\right)\right. \\
& \left.-4 A\left(q^{2} ; m_{\widetilde{u}_{i}}^{2}, m_{\widetilde{d}_{i}}^{2}\right)\right]
\end{aligned}
$$

for left-hand squark doublets, and

$$
\begin{aligned}
& \Pi_{R}^{\gamma \gamma}\left(q^{2}\right)=N_{c} \frac{e^{2}}{9 \pi^{2}} \sum_{i=1,2,3}[-16 A\left(q^{2} ; m_{\widetilde{u}_{R i}}^{2}, m_{\widetilde{u}_{R i}}^{2}\right) \\
&-4 A\left(q^{2} ; m_{\widetilde{d}_{R i}}^{2}, m_{\widetilde{d}_{R i}}^{2}\right) \\
&+\left.8 a\left(m_{\widetilde{u}_{R i}}^{2}\right)+2 a\left(m_{\widetilde{d}_{R i}}^{2}\right)\right], \\
& \Pi_{R}^{Z Z}\left(p^{2}\right)=N_{c} \frac{e^{2}}{9 \pi^{2}} \frac{s^{2}}{c^{2}} \sum_{i=1,2,3}\left[-16 A\left(q^{2} ; m_{\widetilde{u}_{R i}}^{2}, m_{\widetilde{u}_{R i}}^{2}\right)\right. \\
&-4 A\left(q^{2} ; m_{\widetilde{d}_{R i}}^{2}, m_{\widetilde{d}_{R i}}^{2}\right) \\
&\left.+8 a\left(m_{\widetilde{u}_{R i}}^{2}\right)+2 a\left(m_{\widetilde{d}_{R i}}^{2}\right)\right]
\end{aligned}
$$

together with

$$
\begin{aligned}
\Pi_{R}^{\gamma Z}\left(q^{2}\right)=N_{c} \frac{e^{2}}{9 \pi^{2}} \frac{s}{c} \sum_{i=1,2.3}[ & 16 A\left(q^{2} ; m_{\widetilde{u}_{R i}}^{2}, m_{\widetilde{u}_{R i}}^{2}\right) \\
+ & 4 A\left(q^{2} ; m_{\widetilde{d}_{R i}}^{2}, m_{\widetilde{d}_{R i}}^{2}\right) \\
- & \left.8 a\left(m_{\widetilde{u}_{R i}}^{2}\right)-2 a\left(m_{\widetilde{d}_{R i}}^{2}\right)\right], \\
\Pi_{R}^{W W}\left(q^{2}\right) & =0
\end{aligned}
$$

for right-hand squarks. Here, $A\left(p^{2} ; x, y\right)$ and $a(x)$ are oneloop integral functions, which are defined in the appendix. Note that the right-hand sfermions $\widetilde{u}_{R i}, \widetilde{d}_{R i}$ and $\widetilde{e}_{R i}$ have no contributions to the self-energy of $W$ boson. Also, we want to mention that there are four-point vertexes for $W$ bosons from the Lagrangian (1), however they contribute at two-loop.

The effects on $\Pi$ due to FCNC can be taken into account through the reexpression of squark and slepton sectors with their corresponding mass eigenstates. For example, the mixing in mass matrix of $\widetilde{u}_{L}$ and $\widetilde{u}_{R}$ can be diagonalized via a unitary matrix $U\left(\alpha_{1}\right)$ :

$$
\left(\begin{array}{l}
\tilde{u}_{L} \\
\tilde{u}_{R}
\end{array}\right)=\left(\begin{array}{cc}
\cos \alpha_{1} & -\sin \alpha_{1} \\
\sin \alpha_{1} & \cos \alpha_{1}
\end{array}\right)\left(\begin{array}{l}
\tilde{u}_{1} \\
\tilde{u}_{2}
\end{array}\right)
$$

such that the vertexes for sfermions coupled to vector bosons can be read from the mass eigenstates $\widetilde{u}_{1,2}$.

2.3. Slepton Sector. In this sector, the contributions to selfenergy of SM vector bosons stem from the three-generation left-hand sleptons $\tilde{l}_{L i}=\left(\widetilde{v}_{i}, \widetilde{e}_{i}\right)$ and right-hand $\widetilde{e}_{R i}$. In terms of the general expression in (1), we derive that

$$
\begin{aligned}
& \Pi^{\gamma \gamma}\left(q^{2}\right) \\
& =\frac{e^{2}}{\pi^{2}} \sum_{i=1,2,3}\left[-4 A\left(q^{2} ; m_{\widetilde{e}_{i}}^{2}, m_{\widetilde{e}_{i}}^{2}\right)\right. \\
& -4 A\left(q^{2} ; m_{\widetilde{e}_{R i}}^{2}, m_{\widetilde{e}_{R i}}^{2}\right) \\
& \left.+2 a\left(m_{\widetilde{e}_{i}}^{2}\right)+2 a\left(m_{\widetilde{e}_{R}}^{2}\right)\right], \\
& \Pi^{Z Z}\left(p^{2}\right) \\
& =\frac{e^{2}}{\pi^{2}} \sum_{i=1,2,3}\left[\frac{\left(1-2 s^{2}\right)^{2}}{s^{2} c^{2}}\right. \\
& \times\left(-A\left(q^{2} ; m_{\widetilde{e}_{i}}^{2}, m_{\widetilde{e}_{i}}^{2}\right)+\frac{1}{2} a\left(m_{\tilde{e}_{i}}^{2}\right)\right) \\
& +\frac{1}{2 s^{2} c^{2}} a\left(m_{\tilde{v}_{i}}^{2}\right) \\
& -\frac{s^{2}}{c^{2}} \\
& \times\left(4 A\left(q^{2} ; m_{\widetilde{e}_{R i}}^{2}, m_{\widetilde{e}_{R i}}^{2}\right)-2 a\left(m_{\widetilde{e}_{R i}}^{2}\right)\right) \\
& \left.-\frac{s^{2}}{c^{2}} 4 A\left(q^{2} ; m_{\tilde{v}_{i}}^{2}, m_{\tilde{v}_{i}}^{2}\right)\right] \text {, }
\end{aligned}
$$




$$
\begin{aligned}
& \Pi^{\gamma Z}\left(q^{2}\right) \\
& =\frac{e^{2}}{\pi^{2}} \sum_{i=1,2,3}\left[\frac{1-2 s^{2}}{s c}\right. \\
& \times\left(-2 A\left(q^{2} ; m_{\widetilde{e}_{i}}^{2}, m_{\widetilde{e}_{i}}^{2}\right)+a\left(m_{\widetilde{e}_{i}}^{2}\right)\right)
\end{aligned}
$$

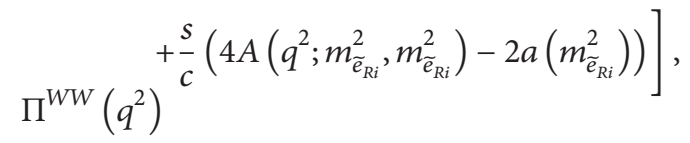

$$
\begin{aligned}
& =\frac{1}{2 \pi^{2}} \frac{e^{2}}{s^{2}} \sum_{i=1,2.3}\left[\left(a\left(m_{\tilde{v}_{i}}^{2}\right)+a\left(m_{\widetilde{e}_{i}}^{2}\right)\right)\right. \\
& \left.-4 A\left(q^{2} ; m_{\tilde{v}_{i}}^{2}, m_{\widetilde{e}_{i}}^{2}\right)\right] .
\end{aligned}
$$

As we have mentioned in the previous subsection, there is no contribution to $\Pi^{W W}$ from the right-hand $\widetilde{e}_{R i}$. The discussion about taking the masses mixings among left- and right-hand sclars is similar to that about (9) via introducing a unitary matrix $U\left(\alpha_{2}\right)$ :

$$
\left(\begin{array}{c}
\widetilde{e}_{L} \\
\widetilde{e}_{R}
\end{array}\right)=\left(\begin{array}{cc}
\cos \alpha_{2} & -\sin \alpha_{2} \\
\sin \alpha_{2} & \cos \alpha_{2}
\end{array}\right)\left(\begin{array}{c}
\widetilde{e}_{1} \\
\tilde{e}_{2}
\end{array}\right)
$$

So far, we have dealt with evaluating the first two graphs in Figure 1, now we proceed to discuss the Higgs sector in which the last graph has to be included.

2.4. Higgs Sector. The calculation of contributions coming from the neutral $A^{0}, H^{0}$ as well as the charged real scalar $H^{ \pm}$ is similar to those of sfermion sector but more involved. One reason is that there is an extra Feynman diagram Figure 1(c) that needs to be considered due to the new couplings with the Higgs VEVs $v_{\mu}$ and $v_{d}$. The other reason is that the expressions when we transform from Higgs gauge eigenstates to their mass eigenstates are complicated.

Consider the following:

$$
\begin{aligned}
& H_{\mu}=\left(\begin{array}{c}
\sin \beta G^{+}+\cos \beta H^{+} \\
\frac{1}{\sqrt{2}} v_{\mu}+\frac{1}{\sqrt{2}} \cos \alpha h^{0}+\frac{1}{\sqrt{2}} \sin \alpha H^{0}+\frac{i}{\sqrt{2}} \sin \beta G^{0}+\frac{i}{\sqrt{2}} \cos \beta A^{0}
\end{array}\right),
\end{aligned}
$$

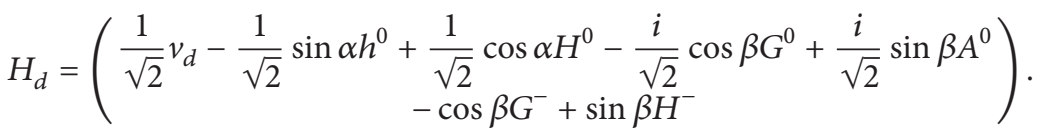

Here $G^{0}$ and $G^{ \pm}$are Nambu-Goldstone modes under the 't Hooft-Feynman gauge. $\tan \beta=v_{\mu} / v_{d}$ and angle $\alpha$ is introduced in the unitary matrix so as to diagonalize the mass matrix of scalars. The mass spectra for scalars in the Higgs sector can be explicitly found in [18]. ing:

Evaluate the Feynman diagrams gives rise to the follow-

$$
\begin{aligned}
& \Pi^{\gamma \gamma}\left(q^{2}\right) \\
& =\frac{e^{2}}{\pi^{2}}\left[-4 A\left(q^{2} ; m_{G^{+}}^{2}, m_{G^{+}}^{2}\right)\right. \\
& -4 A\left(q^{2} ; m_{H^{+}}^{2}, m_{H^{+}}^{2}\right) \\
& +2 a\left(m_{G^{+}}^{2}\right)+2 a\left(m_{H^{+}}^{2}\right) \\
& +\frac{\pi^{2} e^{2}}{2 s^{2}}\left(\sin \beta v_{\mu}+\cos \beta v_{d}\right)^{2} \\
& \left.\times b_{0}\left(q^{2} ; m_{W}^{2}, m_{G^{+}}^{2}\right)\right], \\
& \Pi^{Z Z}\left(q^{2}\right) \\
& =\frac{e^{2}}{4 \pi^{2} s^{2} c^{2}}\left\{a\left(m_{h^{0}}^{2}\right)+a\left(m_{H^{0}}^{2}\right)\right.
\end{aligned}
$$

$$
\begin{aligned}
+ & a\left(m_{G^{0}}^{2}\right)+a\left(m_{A^{0}}^{2}\right) \\
+ & 2\left(1-2 s^{2}\right)^{2} \\
\times & {\left.\left[a\left(m_{G^{+}}^{2}\right)+a\left(m_{H^{+}}^{2}\right)\right]\right\} } \\
+\frac{e^{4}}{4 \pi^{2} s^{4} c^{4}}[ & \left(\cos \alpha v_{\mu}-\sin \alpha v_{d}\right)^{2} \\
& \times b_{0}\left(q^{2} ; m_{Z}^{2}, m_{h^{0}}^{2}\right) \\
& +\left(\sin \alpha v_{\mu}+\cos \alpha v_{d}\right)^{2} \\
& \times b_{0}\left(q^{2} ; m_{Z}^{2}, m_{H^{0}}^{2}\right) \\
& +2 s^{4} c^{2}\left(\sin \beta v_{\mu}+\cos \beta v_{d}\right)^{2} \\
& \left.\times b_{0}\left(q^{2} ; m_{W}^{2}, m_{G^{+}}^{2}\right)\right] \\
-\frac{e^{2}}{\pi^{2} s^{2} c^{2}}\{ & \left(1-2 s^{2}\right)^{2}\left[A\left(q^{2} ; m_{G^{+}}^{2}, m_{G^{+}}^{2}\right)\right. \\
+ & \cos ^{2}(\alpha-\beta)\left[A\left(q^{2} ; m_{h^{0}}^{2}, m_{A^{0}}^{2}\right)\right. \\
& \left.+A\left(q^{2} ; m_{H^{+}}^{2}, m_{H^{+}}^{2}\right)\right] \\
& \\
& \\
& \\
&
\end{aligned}
$$




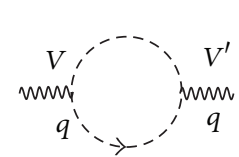

(a)

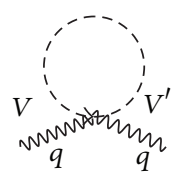

(b)

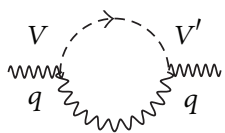

(c)

FIGURE 1: Graphs that contribute to the self-energy of SM gauge bosons due to scalar superpartners.

$$
\begin{aligned}
& +\sin ^{2}(\alpha-\beta) \\
& \left.\times\left[A\left(q^{2} ; m_{h^{0}}^{2}, m_{G^{0}}^{2}\right)+A\left(q^{2} ; m_{H^{0}}^{2}, m_{A^{0}}^{2}\right)\right]\right\},
\end{aligned}
$$

$\Pi^{\gamma Z}\left(q^{2}\right)$

$$
\begin{aligned}
=-\frac{e^{2}\left(1-2 s^{2}\right)}{2 \pi^{2} s c}[ & 2 a\left(m_{G^{+}}^{2}\right)+2 a\left(m_{H^{+}}^{2}\right) \\
& +4 A\left(q^{2} ; m_{G^{+}}^{2}, m_{G^{+}}^{2}\right) \\
& \left.-4 A\left(q^{2} ; m_{H^{+}}^{2}, m_{H^{+}}^{2}\right)\right] \\
-\frac{e^{4}}{2 \pi^{2} s c}\left(\sin \beta v_{\mu}\right. & \left.+\cos \beta v_{d}\right)^{2} b_{0}\left(q^{2} ; m_{W}^{2}, m_{G^{+}}^{2}\right),
\end{aligned}
$$

$\Pi^{W W}\left(q^{2}\right)$

$$
=\frac{e^{2}}{4 \pi^{2} s^{2}}\left[2 a\left(m_{H^{+}}^{2}\right)+2 a\left(m_{G^{+}}^{2}\right)+a\left(m_{H^{0}}^{2}\right)\right.
$$$$
\left.+a\left(m_{A^{0}}^{2}\right)+a\left(m_{G^{0}}^{2}\right)+a\left(m_{h^{0}}^{2}\right)\right]
$$$$
+\frac{e^{4}}{4 \pi^{2} s^{2} c^{2}}\left(\sin \beta v_{\mu}+\cos \beta v_{d}\right)^{2}
$$$$
\times\left[c^{2} b_{0}\left(q^{2} ; 0, m_{G^{+}}^{2}\right)+s^{2} b_{0}\left(q^{2} ; m_{Z}^{2}, m_{G^{+}}^{2}\right)\right]
$$$$
+\frac{e^{4}}{4 \pi^{2} s^{4}}\left[\left(\cos \alpha v_{\mu}-\sin \alpha v_{d}\right)^{2} b_{0}\left(q^{2} ; m_{W}^{2}, m_{h^{0}}^{2}\right)\right.
$$$$
+\left(\sin \alpha v_{\mu}+\cos \alpha v_{d}\right)^{2}
$$$$
\left.\times b_{0}\left(q^{2} ; m_{W}^{2}, m_{H^{0}}^{2}\right)\right]
$$$$
-\frac{e^{2}}{\pi^{2} s^{2}}\left\{\sin ^{2}(\beta-\alpha)\right.
$$

$$
\begin{aligned}
& \times\left[A\left(q^{2} ; m_{h_{0}}^{2}, m_{G^{+}}^{2}\right)+A\left(q^{2} ; m_{H_{0}}^{2}, m_{H^{+}}^{2}\right)\right] \\
& +\cos ^{2}(\alpha+\beta) \\
& \times\left[A\left(q^{2} ; m_{h_{0}}^{2}, m_{H^{+}}^{2}\right)+A\left(q^{2} ; m_{H_{0}}^{2}, m_{G^{+}}^{2}\right)\right] \\
& \left.+A\left(q^{2} ; m_{A_{0}}^{2}, m_{H^{+}}^{2}\right)+A\left(q^{2} ; m_{G_{0}}^{2}, m_{G^{+}}^{2}\right)\right\} .
\end{aligned}
$$

In terms of the corrections arising from the SM, the superpartners' contributions in MSSM can be separated from (14) as

$$
\begin{aligned}
& \Pi^{\gamma \gamma}\left(q^{2}\right)=\frac{e^{2}}{\pi^{2}}\left[-4 A\left(q^{2} ; m_{H^{+}}^{2}, m_{H^{+}}^{2}\right)+2 a\left(m_{H^{+}}^{2}\right)\right], \\
& \Pi^{Z Z}\left(q^{2}\right)=\frac{e^{2}}{4 \pi^{2} s^{2} c^{2}} \\
& \times\left[a\left(m_{H^{0}}^{2}\right)+2 a\left(m_{A^{0}}^{2}\right)+2\left(1-2 s^{2}\right)^{2} a\left(m_{H^{+}}^{2}\right)\right] \\
& +\frac{e^{2}}{\pi^{2} s^{2} c^{4}} m_{W}^{2} \cos ^{2}(\alpha-\beta) \\
& \times\left[b_{0}\left(q^{2} ; m_{Z}^{2}, m_{H^{0}}^{2}\right)-b_{0}\left(q^{2} ; m_{Z}^{2}, m_{H^{0}}^{2}\right)\right] \\
& -\frac{e^{2}}{\pi^{2} s^{2} c^{2}} \\
& \times\left\{\left(1-2 s^{2}\right)^{2} A\left(q^{2} ; m_{H^{+}}^{2}, m_{H^{+}}^{2}\right)\right. \\
& +\cos ^{2}(\alpha-\beta) \\
& \times\left[A\left(q^{2} ; m_{h^{0}}^{2}, m_{A^{0}}^{2}\right)+A\left(q^{2} ; m_{H^{0}}^{2}, m_{G^{0}}^{2}\right)\right] \\
& -\cos ^{2}(\alpha-\beta) A\left(q^{2} ; m_{h^{0}}^{2}, m_{G^{0}}^{2}\right) \\
& \left.+\sin ^{2}(\alpha-\beta) A\left(q^{2} ; m_{H^{0}}^{2}, m_{A^{0}}^{2}\right)\right\}, \\
& \Pi^{\gamma Z}\left(q^{2}\right)=-\frac{e^{2}\left(1-2 s^{2}\right)}{\pi^{2} s c}\left[-2 A\left(q^{2} ; m_{H^{+}}^{2}, m_{H^{+}}^{2}\right)+a\left(m_{H^{+}}^{2}\right)\right], \\
& \Pi^{W W}\left(q^{2}\right)=\frac{e^{2}}{4 \pi^{2} s^{2}}\left[2 a\left(m_{H^{+}}^{2}\right)+a\left(m_{H^{0}}^{2}\right)+a\left(m_{A^{0}}^{2}\right)\right] \\
& +\frac{e^{2}}{\pi^{2} s^{2}} \cos ^{2}(\alpha-\beta) m_{W}^{2} \\
& \times\left[b_{0}\left(q^{2} ; m_{W}^{2}, m_{H^{0}}^{2}\right)-b_{0}\left(q^{2} ; m_{W}^{2}, m_{h^{0}}^{2}\right)\right] \\
& +\frac{e^{2}}{\pi^{2} s^{2}} \cos ^{2}(\alpha-\beta)\left[A\left(q^{2} ; m_{h_{0}}^{2}, m_{G^{+}}^{2}\right)\right. \\
& \text { - } A\left(q^{2} ; m_{h_{0}}^{2}, m_{H^{+}}^{2}\right) \\
& \left.-A\left(q^{2} ; m_{H_{0}}^{2}, m_{G^{+}}^{2}\right)\right]
\end{aligned}
$$




$$
\begin{gathered}
-\frac{e^{2}}{\pi^{2} s^{2}}\left[\sin ^{2}(\alpha-\beta) A\left(q^{2} ; m_{H_{0}}^{2}, m_{H^{+}}^{2}\right)\right. \\
\left.+A\left(q^{2} ; m_{A_{0}}^{2}, m_{H^{+}}^{2}\right)\right] .
\end{gathered}
$$

\section{Preliminary Checks on the Results}

In electroweak theory, there are various observables that can be precisely measured, however, they depend only on three parameters which are composed of the gauge coupling constants parameters $g, g^{\prime}$, and VEV $v$ associated with the scale of spontaneous symmetry breaking. For example, one can define the weak mixing angle as

$$
s_{W}^{2} \equiv 1-\frac{m_{W}^{2}}{m_{Z}^{2}}
$$

Another definition uses

$$
s_{*}^{2} \equiv \frac{g^{\prime 2}}{g^{2}+g^{\prime 2}} .
$$

Alternatively, we do this via the accurately known weakinteraction observables $\alpha, G_{F}$, and $m_{Z}$ :

$$
\sin 2 \theta_{0} \equiv\left(\frac{4 \pi \alpha\left(m_{Z}^{2}\right)}{\sqrt{2} G_{F} m_{Z}^{2}}\right)^{1 / 2}=0.2307 \pm 0.0005
$$

which gives us an accurate standard of reference. The corrections to $\alpha, G_{F}$, and $m_{Z}$ due to quantum effects of new particle states beyond SM will lead to deviation from the reference point. So the measured value can be used to constrain the content of these new particles and estimate the bounds of their masses.

Now we use the weak mixing angle as an example to check our results presented in the previous section. The rational is as follows. The quantum corrections to observables such as $m_{Z}, m_{W}, \alpha$, and $G_{F}$ in low-energy electroweak theory include corrections to the vector boson propagator, vertex, and box corrections. The last two types are usually proportional to the ratio of masses of external (light) fermions over masses of heavy particle states. With the limit that these light masses of SM fermions are set to zero, only the first type is important practically. This type of correction is known as oblique correction, as they enter the low-energy weak interactions only indirectly. The different definitions in (16) to (19) all agree at zero order but receive different radiative corrections. However, the differences, for instance [19],

$$
\begin{aligned}
s_{W}^{2}-s_{*}^{2} \equiv & -\frac{c^{2}}{m_{W}^{2}}\left[\Pi^{W W}\left(m_{W}^{2}\right)-c^{2} \Pi^{Z Z}\left(m_{Z}^{2}\right)\right] \\
& +\frac{s c^{3}}{m_{W}^{2}} \Pi^{\gamma Z}\left(m_{Z}^{2}\right)
\end{aligned}
$$

due to the radiative corrections must be finite and are free of ultraviolet divergence.
For the part of slepton sector, substituting (10) into (19) gives

$$
\begin{aligned}
s_{W}^{2}-s_{*}^{2}=\frac{c^{2} e^{2}}{\pi^{2} s^{2} m_{W}^{2}}[ & -\left(1-2 s^{2}\right) A\left(m_{Z}^{2} ; m_{\widetilde{e}_{i}}^{2}, m_{\widetilde{e}_{i}}^{2}\right) \\
& -A\left(m_{Z}^{2} ; m_{\widetilde{v}_{i}}^{2}, m_{\tilde{v}_{i}}^{2}\right) \\
& \left.+2 A\left(m_{W}^{2} ; m_{\widetilde{e}_{i}}^{2}, m_{\widetilde{v}_{i}}^{2}\right)-s^{2} a\left(m_{\widetilde{e}_{i}}^{2}\right)\right]
\end{aligned}
$$

for each generation of left-hand sleptons, which then gives us the part of divergence that is proportional to

$$
\begin{aligned}
& -\left(1-2 s^{2}\right)\left(\frac{1}{2} m_{\widetilde{e}_{i}}^{2}-\frac{1}{12} m_{Z}^{2}\right) \\
& -\left(\frac{1}{2} m_{\widetilde{v}_{i}}^{2}-\frac{1}{12} m_{Z}^{2}\right) \\
& +2\left(\frac{1}{4} m_{\widetilde{e}_{i}}^{2}+\frac{1}{4} m_{\widetilde{v}_{i}}^{2}-\frac{1}{12} m_{W}^{2}-s^{2} m_{\widetilde{e}_{i}}^{2}\right)=0 .
\end{aligned}
$$

For each generation of right-hand slepton, we find that the contribution to the deference in (19) vanishes.

Now we proceed to examine the results in the squark sector. Substituting (5) into (19) yields

$$
\begin{aligned}
s_{W}^{2}-s_{*}^{2}=\frac{e^{2} c^{2}}{\pi^{2} s^{2} m_{W}^{2}}[ & -\left(1-\frac{3}{4} s^{2}\right) A\left(m_{Z}^{2} ; m_{\widetilde{u}_{i}}^{2}, m_{\widetilde{u}_{i}}^{2}\right) \\
& -\frac{2}{3} s^{2} a\left(m_{\widetilde{u}_{i}}^{2}\right)-\frac{1}{3} s^{2} a\left(m_{\tilde{d}_{i}}^{2}\right) \\
& -\left(1-\frac{2}{3} s^{2}\right) A\left(m_{Z}^{2} ; m_{\widetilde{d}_{i}}^{2}, m_{\widetilde{d}_{i}}^{2}\right) \\
& \left.+2 A\left(m_{W}^{2} ; m_{\widetilde{u}_{i}}^{2}, m_{\tilde{d}_{i}}^{2}\right)\right]
\end{aligned}
$$

for the left-hand squarks, from which the part of divergence is proportional to

$$
\begin{aligned}
& -\left(1-\frac{3}{4} s^{2}\right)\left(\frac{1}{2} m_{\widetilde{u}_{i}}^{2}-\frac{1}{12} m_{Z}^{2}\right) \\
& -\frac{2}{3} s^{2} m_{\widetilde{u}_{i}}^{2}-\frac{1}{3} s^{2} m_{\widetilde{d}_{i}}^{2} \\
& -\left(1-\frac{2}{3} s^{2}\right)\left(\frac{1}{2} m_{\widetilde{d}_{i}}^{2}-\frac{1}{12} m_{Z}^{2}\right) \\
& +2\left(\frac{1}{4} m_{\widetilde{u}_{i}}^{2}+\frac{1}{4} m_{\widetilde{d}_{i}}^{2}-\frac{1}{12} m_{Z}^{2}\right)=0 .
\end{aligned}
$$

For the part of Higgs sector, substituting (14) into (19) gives that the divergent parts in (19) are composed of those arising from $a(x), A(x)$, and $b_{0}$ functions. It turns out that the contribution from $a(x)$ function is proportional to $-\left(e^{2} c^{2} / \pi^{2} m_{W}^{2}\right)\left(m_{G^{+}}^{2}+m_{H^{+}}^{2}\right)$, which exactly cancels the part from $A$ function. And the divergent parts in $b_{0}$ function cancel themselves. 


\section{4. $S, T$, and $U$ in the MSSM}

In this section, we derive a set of parameters which estimate the oblique corrections to precise electroweak observables. These parameters are known as $S, T$, and $U$ in the literature $[3,4]$. As a byproduct we also use the property that these parameters are finite values to examine the results shown in Section 2, independently of what we have done in Section 3. The dependence of $S, T$, and $U$ parameters on $\Pi^{I J}\left(p^{2}\right)$ can be perturbatively expanded in powers of the external momentum squared. It turns out that these corrections are quite simple $[3,4]$ :

$$
\begin{aligned}
S \equiv & -\frac{16 \pi}{e^{2}} s c\left[s c \Pi^{\gamma \gamma^{\prime}}(0)-s c \Pi^{Z Z^{\prime}}(0)+\left(c^{2}-s^{2}\right) \Pi^{\gamma Z^{\prime}}(0)\right] \\
T \equiv & \frac{4 \pi}{e^{2}}\left[\frac{\Pi^{W W}(0)}{m_{W}^{2}}-\frac{\Pi^{Z Z}(0)}{m_{Z}^{2}}-\frac{2 s}{c} \frac{\Pi^{\gamma Z}(0)}{m_{Z}^{2}}\right], \\
U \equiv & \frac{16 \pi s^{2}}{e^{2}} \\
& \times\left[\Pi^{W W^{\prime}}(0)-c^{2} \Pi^{Z Z^{\prime}}(0)-s^{2} \Pi^{\gamma \gamma^{\prime}}(0)-2 c s \Pi^{\gamma Z^{\prime}}(0)\right],
\end{aligned}
$$

where

$$
\Pi^{I J^{\prime}}(0)=\left.\frac{d^{2} \Pi^{I J}}{d p^{2}}\right|_{p^{2}=0},
$$

$\Pi^{I J}$ is the part with metric as the coefficient in $\Pi_{\mu \nu}^{I J}=g_{\mu \nu} \Pi^{I J}+$ $\cdots$.

In this paper, we consider the calculations of the oblique corrections in MSSM, which are composed of four parts given a specific vacuum polarization:

$$
\Pi_{\text {SUSY }}=\Pi_{\widetilde{S}}+\Pi_{\tilde{l}}+\Pi_{H}+\Pi_{N C} .
$$

We derive the first three parts in (26) in this paper, and will explore the last part in the future [15].

Firstly, the slepton sector gives

$$
\begin{aligned}
& S_{\tilde{l}}=\frac{1}{12 \pi} \sum_{i=1}^{3} \ln \frac{m_{\widetilde{v}_{i}}^{2}}{m_{\widetilde{e}_{i}}^{2}} \\
& T_{\widetilde{l}}=\frac{1}{16 \pi s^{2} m_{W}^{2}} \sum_{i=1}^{3}\left[m_{\tilde{v}_{i}}^{2}+m_{\widetilde{e}_{i}}^{2}-\frac{2 m_{\tilde{e}_{i}}^{2} m_{\widetilde{v}_{i}}^{2} \ln \left(m_{\tilde{v}_{i}}^{2} / m_{\widetilde{e}_{i}}^{2}\right)}{m_{\widetilde{v}_{i}}^{2}-m_{\widetilde{e}_{i}}^{2}}\right] \text {, } \\
& U_{\tilde{l}}=\frac{1}{\pi} \sum_{i=1}^{3}\left[\frac{m_{\tilde{e}_{i}}^{2}}{3} b_{0}^{\prime}\left(0, m_{\widetilde{e}_{i}}^{2}, m_{\widetilde{e}_{i}}^{2}\right)\right. \\
& -\frac{m_{\tilde{e}_{i}}^{2}+m_{\tilde{v}_{i}}^{2}}{6} b_{0}^{\prime}\left(0, m_{\tilde{e}_{i}}^{2}, m_{\tilde{v}_{i}}^{2}\right) \\
& +\frac{\left(m_{\widetilde{e}_{i}}^{2}-m_{\tilde{v}_{i}}^{2}\right)^{2}}{24} b_{0}^{\prime \prime}\left(0, m_{\widetilde{e}_{i}}^{2}, m_{\tilde{v}_{i}}^{2}\right) \\
& \left.+\frac{1}{12} f\left(m_{\widetilde{e}_{i}}^{2}, m_{\widetilde{v}_{i}}^{2}\right)-\frac{1}{12} f\left(m_{\widetilde{e}_{i}}^{2}, m_{\widetilde{e}_{i}}^{2}\right)\right] \text {. }
\end{aligned}
$$

For the squark sector, we have

$$
\begin{aligned}
& S_{\widetilde{S}}=\frac{1}{12 \pi} \sum_{i=1}^{3} \ln \frac{m_{\widetilde{d}_{i}}^{2}}{m_{\widetilde{u}_{i}}^{2}} \\
& T_{\widetilde{s}}=\frac{3}{16 \pi s^{2} m_{W}^{2}} \\
& \times \sum_{i=1}^{3}\left[m_{\widetilde{u}_{i}}^{2}+m_{\widetilde{d}_{i}}^{2}-\frac{2 m_{\tilde{u}_{i}}^{2} m_{\widetilde{d}_{i}}^{2} \ln \left(m_{\widetilde{u}_{i}}^{2} / m_{\widetilde{d}_{i}}^{2}\right)}{m_{\widetilde{u}_{i}}^{2}-m_{\widetilde{d}_{i}}^{2}}\right], \\
& U_{\widetilde{S}}=\sum_{i=1}^{3} \frac{1}{\pi}\left[m_{\widetilde{u}_{i}}^{2} b_{0}^{\prime}\left(0, m_{\widetilde{u}_{i}}^{2}, m_{\widetilde{u}_{i}}^{2}\right)+m_{\tilde{d}_{i}}^{2} b_{0}^{\prime}\left(0, m_{\widetilde{d}_{i}}^{2}, m_{\widetilde{d}_{i}}^{2}\right)\right. \\
& -\left(m_{\widetilde{u}_{i}}^{2}+m_{\tilde{d}_{i}}^{2}\right) b_{0}^{\prime}\left(0, m_{\tilde{u}_{i}}^{2}, m_{\tilde{d}_{i}}^{2}\right) \\
& +\frac{1}{2} f\left(m_{\widetilde{u}_{i}}^{2}, m_{\tilde{d}_{i}}^{2}\right)-\frac{1}{4} f\left(m_{\widetilde{u}_{i}}^{2}, m_{\widetilde{u}_{i}}^{2}\right) \\
& \left.-\frac{1}{4} f\left(m_{\tilde{d}_{i}}^{2}, m_{\tilde{d}_{i}}^{2}\right)\right]
\end{aligned}
$$

with the finite function $f(x, y)$ is defined as

$$
f(x, y)=\frac{1}{x-y}(x \ln x-y \ln y) .
$$

Note that $b_{0}^{\prime}$ and $b_{0}^{\prime \prime}$ in (27) to (28) are given in appendix, which are also finite.

Finally, the Higgs sector yields

$$
\begin{aligned}
\pi S_{H}= & h\left(m_{H^{+}}^{2}, m_{H^{+}}^{2}\right)-\sin ^{2}(\alpha-\beta) h\left(m_{A_{0}}^{2}, m_{H_{0}}^{2}\right) \\
& +\cos ^{2}(\alpha-\beta) \\
& \times\left[h\left(m_{h_{0}}^{2}, m_{G_{0}}^{2}\right)-h\left(m_{H_{0}}^{2}, m_{G_{0}}^{2}\right)-h\left(m_{h_{0}}^{2}, m_{A_{0}}^{2}\right)\right] \\
& -\frac{m_{W}^{2}}{c^{2}} \cos ^{2}(\alpha-\beta) \\
& \times\left[b_{0}^{\prime}\left(0 ; m_{Z}^{2}, m_{h_{0}}^{2}\right)-b_{0}^{\prime}\left(0 ; m_{Z}^{2}, m_{H_{0}}^{2}\right)\right],
\end{aligned}
$$




$$
\begin{aligned}
& \pi T_{H}=\frac{1}{4 s^{2} m_{W}^{2}} \\
& \times\left\{m_{H^{+}}^{2} \ln m_{H^{+}}^{2}+\cos ^{2}(\alpha-\beta)\right. \\
& \times\left[m_{W}^{2}\left(f\left(m_{W}^{2}, m_{H_{0}}^{2}\right)-f\left(m_{W}^{2}, m_{h_{0}}^{2}\right)\right)\right. \\
& \left.+m_{Z}^{2}\left(f\left(m_{Z}^{2}, m_{h_{0}}^{2}\right)-f\left(m_{Z}^{2}, m_{H_{0}}^{2}\right)\right)\right] \\
& +\cos ^{2}(\alpha-\beta) \\
& \times\left[g\left(m_{h_{0}}^{2}, m_{G^{+}}^{2}\right)\right. \\
& +g\left(m_{h_{0}}^{2}, m_{A^{0}}^{2}\right) \\
& +g\left(m_{H_{0}}^{2}, m_{G^{0}}^{2}\right) \\
& -g\left(m_{h_{0}}^{2}, m_{H^{+}}^{2}\right) \\
& \left.-g\left(m_{H_{0}}^{2}, m_{G^{+}}^{2}\right)-g\left(m_{h_{0}}^{2}, m_{G^{0}}^{2}\right)\right] \\
& -\sin ^{2}(\alpha-\beta) g\left(m_{H_{0}}^{2}, m_{H^{+}}^{2}\right) \\
& +\sin ^{2}(\alpha-\beta) g\left(m_{A_{0}}^{2}, m_{H^{0}}^{2}\right) \\
& \left.-g\left(m_{A_{0}}^{2}, m_{H^{0}}^{2}\right)\right\}
\end{aligned}
$$

together with

$$
\begin{aligned}
\pi U_{H}= & h\left(m_{H_{+}}^{2}, m_{H^{+}}^{2}\right)-h\left(m_{A_{0}}^{2}, m_{H^{0}}^{2}\right) \\
& -\cos ^{2}(\alpha-\beta) m_{W}^{2} \\
\times & {\left[b_{0}^{\prime}\left(0 ; m_{W}^{2}, m_{H_{0}}^{2}\right)-b_{0}^{\prime}\left(0 ; m_{W}^{2}, m_{h_{0}}^{2}\right)\right] } \\
+ & \cos ^{2}(\alpha-\beta) m_{Z}^{2} \\
\times & {\left[b_{0}^{\prime}\left(0 ; m_{Z}^{2}, m_{h_{0}}^{2}\right)-b_{0}^{\prime}\left(0 ; m_{Z}^{2}, m_{H_{0}}^{2}\right)\right] } \\
+ & \cos ^{2}(\alpha-\beta) \\
\times & {\left[h\left(m_{h_{0}}^{2}, m_{G^{+}}^{2}\right)-h\left(m_{h_{0}}^{2}, m_{H^{+}}^{2}\right)\right.} \\
& \quad-h\left(m_{H_{0}}^{2}, m_{G^{+}}^{2}\right)+h\left(m_{h_{0}}^{2}, m_{A^{0}}^{2}\right) \\
& \left.\quad-h\left(m_{h_{0}}^{2}, m_{G^{0}}^{2}\right)+h\left(m_{G_{0}}^{2}, m_{H^{0}}^{2}\right)\right] \\
& -\sin ^{2}(\alpha-\beta)\left[h\left(m_{H_{0}}^{2}, m_{H^{+}}^{2}\right)-h\left(m_{A_{0}}^{2}, m_{H^{0}}^{2}\right)\right]
\end{aligned}
$$

with the finite functions $g(x, y)$ and $h(x, y)$ defined as

$$
\begin{aligned}
g(x, y)= & \frac{1}{12}(x \ln x+y \ln y) \\
& +\frac{x+y}{6}[-1+f(x, y)] \\
& +\frac{1}{24}\left(x+y-\frac{2 x y}{x-y} \ln \frac{x}{y}\right), \\
h(x, y)= & \frac{1}{6}(x+y) b_{0}^{\prime}(0 ; x, y) \\
& -\frac{1}{24}(x-y)^{2} b_{0}^{\prime \prime}(0 ; x, y)-\frac{1}{12} f(x, y) .
\end{aligned}
$$

Note that the combination $\cos (\alpha-\beta)$ is given by

$$
\cos ^{2}(\alpha-\beta)=\frac{m_{h}^{2}}{m_{A}^{2}} \frac{m_{Z}^{2}-m_{h}^{2}}{m_{Z}^{2}+m_{A}^{2}-2 m_{h}^{2}} .
$$

Here, a few comments are in order for the results (27) to (31). First, it is obvious that each part in MSSM contributes to the finite $S, T$, and $U$ values as required. There are some constants terms in the expression of $S, T$, and $U$ parameters, which naively violate the property that large superpartner masses (compared with $m_{Z}$ ) leading to the conclusion that the theory decouples from the SM. But expanding the relevant functions we have defined implies that these constants are cancelled and the conclusion is restored. Second, from (30) to (31) it seems that the SM contributions has not been separated from the MSSM contributions due to some terms involved with SM fields. But it is crucial to notice that these terms are multiplied by a factor $\cos ^{2}(\alpha-\beta)$, as given by (33). Under the limit that the superpartners decouple from SM, which results in $\alpha=\beta$, the results in (30) to (31), are reduced to pure MSSM ones. Finally, unlike in the case of SM, where the sensitivity of these parameters to the SM Higgs mass $m_{h}$ is logarithmic, the results in (30) to (32) demonstrate that this is not strictly true in the MSSM anymore. According to the simulations about $S, T$, and $U$ shown in [14], the sensitivity of these parameters to the mass of Higgs boson can be either stronger or weaker, which depends on choices of parameters left in the Higgs sector. Overall, the discrepancy is not so significant as one expects roughly.

Under the assumption that the breaking of $S U(2)$ symmetry is weak, that is, $\Delta_{q_{i}}=m_{\widetilde{e}_{i}}^{2}-m_{\widetilde{v}_{i}}^{2} \ll m_{\widetilde{v}_{i}}^{2}, m_{\widetilde{e}_{i}}^{2}$ and $\Delta_{S_{i}}=m_{\widetilde{d}_{i}}^{2}-m_{\widetilde{u}_{i}}^{2} \ll m_{\widetilde{u}_{i}}^{2}, m_{\tilde{d}_{i}}^{2}$, we find

$$
\begin{aligned}
& S_{\tilde{q}} \longrightarrow \frac{1}{12 \pi} \sum_{i=1}^{3} \frac{\Delta_{S_{i}}}{m_{\tilde{d}_{i}}^{2}}, \\
& T_{\widetilde{q}} \longrightarrow-\frac{3}{16 \pi s^{2}} \sum_{i=1}^{3} \frac{\Delta_{S_{i}}}{m_{W}^{2}},
\end{aligned}
$$




$$
\begin{gathered}
U_{\widetilde{q}} \longrightarrow-\frac{1}{4 \pi} \sum_{i=1}^{3} \frac{\Delta_{S_{i}}}{m_{\widetilde{d}_{i}}^{2}}, \\
S_{\widetilde{l}} \longrightarrow-\frac{1}{12 \pi} \sum_{i=1}^{3} \frac{\Delta_{q_{i}}}{m_{\widetilde{e}_{i}}^{2}}, \\
T_{\widetilde{l}} \longrightarrow-\frac{1}{16 \pi s^{2}} \sum_{i=1}^{3} \frac{\Delta_{q_{i}}}{m_{W}^{2}}, \\
U_{\tilde{l}} \longrightarrow-\frac{1}{24 \pi} \sum_{i=1}^{3} \frac{\Delta q_{q_{i}}}{m_{\widetilde{e}_{i}}^{2}} .
\end{gathered}
$$

From (34), one sees that both in the squark and slepton sector the relative ratio $|S / U|$ is approximatively around the unity, while their values relative to $T$ depend on the ratios of $m^{2} / m_{\tilde{d}_{i}}^{2}$ and $m^{2} / m_{\widetilde{e}_{i}}^{2}$.

\section{Discussions and Conclusions}

In this paper, we have revisited the oblique corrections in the context of the MSSM. The motivation for exploring these contributions is quite clear since they are useful to interpret the latest LHC data about the Higgs mass and bounds on superpartner masses. (Note that there is no need to consider the gauge invariance of these contributions once again, as this problem in the SM contributions has been properly treated [20-22].) Thus, we reconsider the theoretic calculations under 't Hooft-Feynman gauge, with the bosonic part, as the first step towards the complete answer.

The results presented in this paper are checked by two examinations. In one examination, we directly confront our results to the finite radiative correction to the deference $s_{W}^{2}-$ $s_{*}^{2}$ in each sector. It shows that the individual contribution deriving from each sector indeed respects this property. The other examination is by using the property that the $S, T$, and $U$ parameters are finite. We also verify the expectation.

In summary, our results about one-loop self energies are found to exactly agree with $[6,16]$. (The formulae presented in [16] take the SM part into account, however, they do not include the contribution arising from the pure gauge part. As we missed this point, we made the claims about disagreement with [16] in the previous version of this paper.) Nevertheless, the $S, T$, and $U$ parameters do not match with those of [11], which cannot be explained by the possible difference of renormalization scheme performed. In particular, our results do not take the scalar mass mixing among left and right hand into account, and therefore correspond to the simple case with vanishing mass mixing. Taking this difference into account and comparing our results with [11], one finds the following.

(i) The $S$ and $U$ parameters arising from squark and slepton sector do not agree.

(ii) The $T$ parameter in (27) and (28) agrees with [11].
We leave the study of oblique corrections arising from the fermions, that is, the neutralinos and charginos, elsewhere [15].

\section{Appendix}

\section{One-Loop Integrals}

Two-point functions $A\left(p^{2} ; x, y\right), a(x)$, and $b_{0}\left(p^{2} ; x, y\right)$ are defined as the integrals [23-25]

$$
\begin{gathered}
\mu^{\epsilon} \int \frac{d^{n} k}{(2 \pi)^{n}} \frac{i}{k^{2}-m^{2}}=\frac{1}{(4 \pi)^{2}} a\left(m^{2}\right), \\
\mu^{\epsilon} \int \frac{d^{n} k}{(2 \pi)^{n}} \frac{i k_{\mu} k_{v}}{\left[k^{2}-m_{1}^{2}\right]\left[(k+p)^{2}-m_{2}^{2}\right]} \\
=\frac{1}{(4 \pi)^{2}}\left[g_{\mu \nu} A\left(p^{2} ; m_{1}^{2}, m_{2}^{2}\right)+\cdots\right], \\
\mu^{\epsilon} \int \frac{d^{n} k}{(2 \pi)^{n}} \frac{i}{\left[k^{2}-m_{1}^{2}\right]\left[(k+p)^{2}-m_{2}^{2}\right]} \\
=\frac{1}{(4 \pi)^{2}} b_{0}\left(p^{2} ; m_{1}^{2}, m_{2}^{2}\right),
\end{gathered}
$$

respectively, where $p^{2}$ is the external line vector boson momentum squared. Here, we have ignored terms that are irrelevant for discussions in the definition of $A\left(p^{2} ; x, y\right)$. Explicitly,

$$
\begin{gathered}
a(x)=-\eta x+a_{F}(x), \\
b_{0}\left(p^{2} ; x, y\right)=-\eta+b_{F}\left(p^{2} ; x, y\right),
\end{gathered}
$$

where the divergent factor in these functions is carried by $\eta$ as follows:

$$
\eta=\frac{1}{\epsilon}+\ln (4 \pi)-\gamma_{E}
$$

Here, $d=4-2 \epsilon$ and $\mu$ is the RG scale. The finite parts $a_{F}$ and $b_{F}$ in (A.2) are given by

$$
\begin{gathered}
a_{F}(x)=x\left(-1+\ln \frac{x}{\mu^{2}}\right) \\
b_{F}\left(p^{2} ; x, y\right)=\int_{0}^{1} d t \ln \frac{t x+(1-t) y-t(1-t) p^{2}}{\mu^{2}}
\end{gathered}
$$


respectively. And

$$
\begin{aligned}
A\left(p^{2} ; x, y\right)= & \left(\frac{p^{2}}{12}-\frac{x+y}{4}\right) \eta \\
& +\frac{1}{12}\left[a_{F}(x)+a_{F}(y)\right] \\
& +\frac{1}{6}\left(x+y-\frac{p^{2}}{2}\right) b_{F}\left(p^{2} ; x, y\right) \\
& +\frac{x-y}{12 p^{2}} \\
& \times\left[a_{F}(x)-a_{F}(y)-(x-y) b_{F}\left(p^{2} ; x, y\right)\right] \\
& -\frac{1}{6}\left(x+y-\frac{p^{2}}{3}\right) .
\end{aligned}
$$

Note that the $A\left(p^{2} ; x, y\right)$ and $b_{0}\left(p^{2} ; x, y\right)$ functions are symmetric in $x$ and $y$.

It is also useful to notice some descents of $b_{0}$ functions:

$$
\begin{gathered}
b_{0}^{\prime}(0 ; x, y)=-\frac{1}{2} \frac{x+y}{(x-y)^{2}}+\frac{x y}{(x-y)^{3}} \ln \frac{x}{y}, \\
b_{0}^{\prime \prime}(0 ; x, y)=\frac{x^{3}+9 x^{2} y-9 x y^{2}-6 x y(x+y) \ln (x / y)-y^{3}}{3(x-y)^{5}} .
\end{gathered}
$$

\section{Conflict of Interests}

The authors do not have a direct financial relation with any commercial identity mentioned in the paper that might lead to a conflict of interests for any of the authors.

\section{Acknowledgment}

Sibo Zheng would like to thank Professor Ken-ichi Hikasa for his correspondence. The work is supported in part by National Natural Science Foundation of China with Grant no. 11247031.

\section{References}

[1] F. Gianotti and ATLAS Collaboration, in Proceedings of the CERN, July 2012.

[2] J. Incandela and CMS Collaboration, in Proceedings of the CERN, July 2012.

[3] G. Aad, T. Abajyan, B. Abbott et al., "Observation of a new particle in the search for the Standard Model Higgs boson with the ATLAS detector at the LHC," Physics Letters B, vol. 716, no. 1, pp. 1-29, 2012.

[4] M. E. Peskin and T. Takeuchi, "New constraint on a strongly interacting Higgs sector," Physical Review Letters, vol. 65, no. 8, pp. 964-967, 1990.

[5] K. Hagiwara, S. Matsumoto, D. Haidt, and C. S. Kim, "A novel approach to confront electroweak data and theory," Zeitschrift für Physik C, vol. 64, no. 4, pp. 559-620, 1994.
[6] D. M. Pierce, J. A. Bagger, K. T. Matchev, and R. Zhang, "Precision corrections in the minimal supersymmetric standard model," Nuclear Physics B, vol. 491, pp. 3-67, 1997.

[7] S. Chatrchyan, V. Khachatryan, A. M. Sirunyan et al., "Observation of a new boson at a mass of $125 \mathrm{GeV}$ with the CMS experiment at the LHC," Physics Letters B, vol. 716, pp. 30-61, 2012.

[8] The ATLAS Collaboration, "Search for supersymmetry in events with four or more leptons and missing transverse momentum in pp collisions at $\sqrt{s}=7 \mathrm{TeV}$ with the ATLAS detector," in Proceedings of the Association for Tourism and Leisure Education (ATLAS '12), 2012.

[9] The CMS Collaboration, "Search for supersymmetry in allhadronic events with missing energy," in Proceedings of the CMS PAS SUS

[10] K. Hagiwara, D. Haidt, and S. Matsumoto, "Analysis of electroweak precision data and prospects for future improvements," European Physical Journal C, vol. 2, no. 1, pp. 95-122, 1998.

[11] A. Dobado, M. J. Herrero, and S. Peñaranda, "Decoupling of supersymmetric particles," European Physical Journal C, vol. 7, no. 2, pp. 313-339, 1999.

[12] A. Dobado, M. J. Herrero, and S. Peñaranda, "The SM as the quantum low-energy effective theory of the MSSM," European Physical Journal C, vol. 12, no. 4, pp. 673-700, 2000.

[13] A. Dobado, M. J. Herrero, and S. Peñaranda, "The Higgs sector of the MSSM in the decoupling limit," European Physical Journal C, vol. 17, no. 3, pp. 487-500, 2000.

[14] S. Zheng and Y. Yu, "Electroweak precision tests on the MSSM and NMSSM constrained at the LHC," Journal of High Energy Physics, vol. 1308, p. 031, 2013.

[15] Y. Yu and S. Zheng, "Oblique corrections in the mssm at one loop ii: fermions," Modern Physics Letters A, vol. 28, Article ID 1250238, 10 pages, 2013.

[16] M. Drees, K. Hagiwara, and A. Yamada, "Process-independent radiative corrections in the minimal supersymmetric standard model," Physical Review D, vol. 45, no. 5, pp. 1725-1743, 1992.

[17] J. F. Gunion, H. E. Haber, G. L. Kane, and S. Dawson, The Higgs Hunter's Guide, vol. 80 of Frontiers of Physics, 2000.

[18] S. P. Martin, "Asupersymmetry primer," http://arxiv.org/abs/ hepph/9709356.

[19] M. E. Peskin and D. V. Schroeder, An Introduction to Quantum Field Theory, Addison-Wesley, Reading, Mass, USA, 1995.

[20] G. Degrassi and A. Sirlin, "Gauge dependence of basic electroweak corrections of the Standard Model," Nuclear Physics B, vol. 383, no. 1-2, pp. 73-92, 1992.

[21] G. Degrassi and A. Sirlin, "Gauge-invariant self-energies and vertex parts of the standard model in the pinch technique framework," Physical Review D, vol. 46, no. 7, pp. 3104-3116, 1992.

[22] G. Degrassi, B. A. Kniehl, and A. Sirlin, "Gauge-invariant formulation of theS, T, and U parameters," Physical Review D, vol. 48, no. 9, pp. R3963-R3966, 1993.

[23] S. Pokorski, Gauge Field Theories, Cambridge University Press, Cambridge, UK, 2nd edition, 2000.

[24] G. 't Hooft and M. Veltman, "Scalar one-loop integrals," Nuclear Physics B, vol. 153, no. 3-4, pp. 365-401, 1979.

[25] G. Passarino and M. Veltman, "One-loop corrections for e+eannihilation into $\mu+\mu$ - in the Weinberg model," Nuclear Physics $B$, vol. 160, no. 1, pp. 151-207, 1979. 

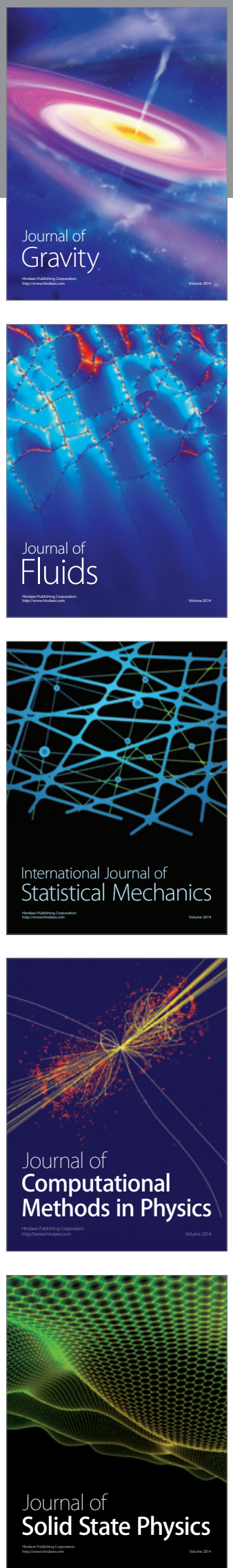

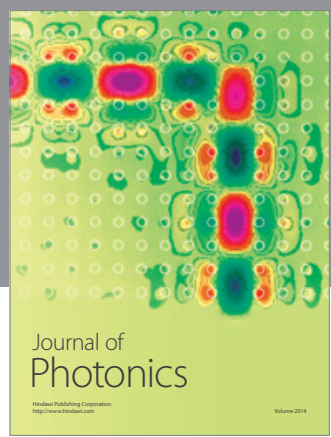

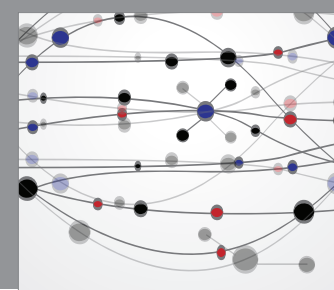

The Scientific World Journal

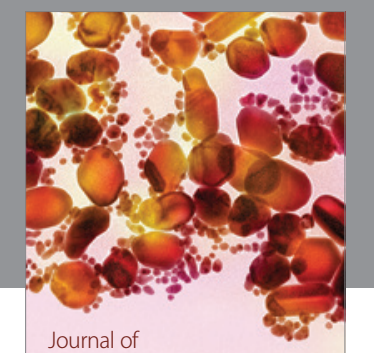

Soft Matter
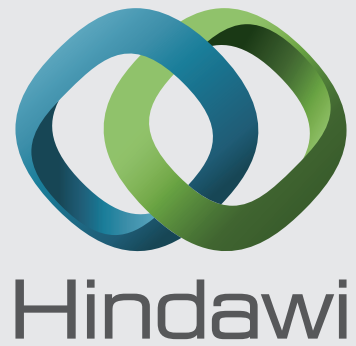

Submit your manuscripts at

http://www.hindawi.com
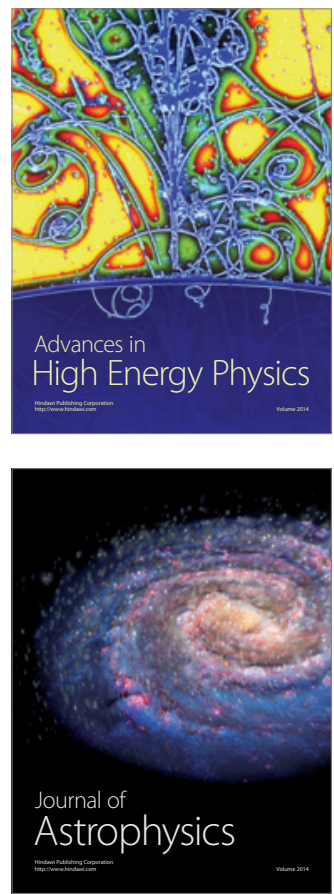
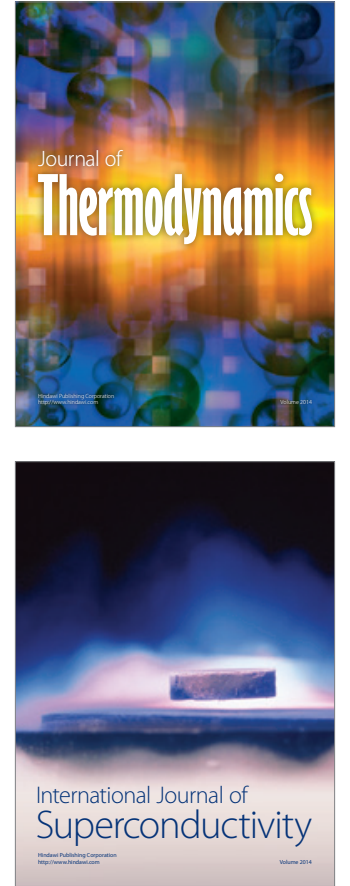
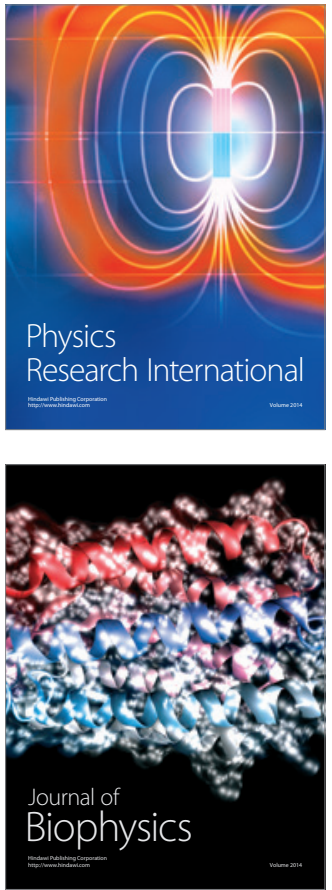
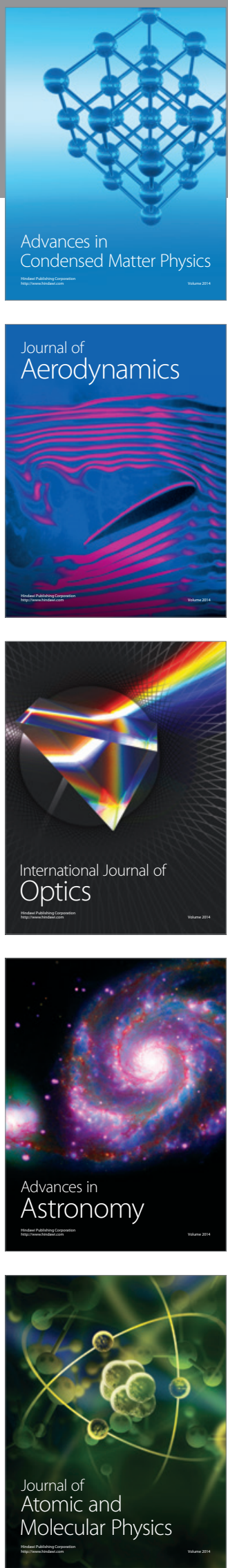